\title{
Nonlinear Hydroelastic Interaction among a Floating Elastic Plate, Water Waves, and Exponential Shear Currents
}

\author{
Ping Wang $\left(\mathbb{D}^{1}\right.$, Yongyan Wang $\mathbb{D}^{2}$, and Xintai Huo ${ }^{1}$ \\ ${ }^{1}$ School of Mathematics and Physics, Qingdao University of Science and Technology, Songling Road, Qingdao 266061, China \\ ${ }^{2}$ College of Electromechanical Engineering, Qingdao University of Science and Technology, Songling Road, Qingdao 266061, China
}

Correspondence should be addressed to Ping Wang; pingwang2003@126.com

Received 12 May 2020; Revised 8 July 2020; Accepted 30 July 2020; Published 26 August 2020

Guest Editor: Yu-Hao Sun

Copyright (c) 2020 Ping Wang et al. This is an open access article distributed under the Creative Commons Attribution License, which permits unrestricted use, distribution, and reproduction in any medium, provided the original work is properly cited.

\begin{abstract}
Nonlinear hydroelastic interaction among a floating elastic plate, a train of deepwater waves, and a current which decays exponentially with depth is studied analytically. We introduce a stream function to obtain the governing equation with the dynamic boundary condition expressing a balance among the hydrodynamic, the shear currents, elastic, and inertial forces. We use the Dubreil-Jacotin transformation to reformulate the unknown free surface as a fixed location in the calculations. The convergent analytical series solutions for the floating plate deflection are obtained with the aid of the homotopy analysis method (HAM). The effects of the shear current are discussed in detail. It is found that the phase speed decreases with the increase of the vorticity parameter in the opposing current, while the phase speed increases with the increase of the vorticity parameter in the aiding current. Larger vorticity tends to increase the horizontal velocity. In the opposing current, the horizontal velocity under the wave crest delays more quickly as the depth increases than that of waves under the wave trough, while in the aiding current case, there is the opposite effect. Furthermore, the larger vorticity can sharpen the hydroelastic wave crest and smooth the trough on an opposing current, while it produces an opposite effect on an aiding current.
\end{abstract}

\section{Introduction}

Hydroelastic interaction between a floating deformable plate and water flows has been a long-standing and hot issue under the rapidly growing demand for exploiting ocean resources and utilizing marine space. For example, hydroelastic interaction has become an indispensable factor during designing a very large floating structure (VLFS) as a storage facility, a mobile offshore base, or even an aircraft airport, which also is available to analyze the floating ice sheet in the polar region, ice-breaking with air-cushioned vehicles, and marine climate.

There is an extensive literature on the theory of nonlinear hydroelastic interaction between a floating elastic plate and the water waves. Most of the relevant research is under the hypothesis that there is no current in oceanic environments, such as Refs. [1-6]. In fact, there are various reasons such as wind, thermal, earth rotation, tidal effects, the vertical variation of water salinity, and temperature which frequently generate ocean currents. Some authors have considered the problem of hydroelastic waves propagating on a current. Schulkes et al. [7] first built the governing equation with the boundary conditions to research the effect of the uniform flow in the underlying water on a floating ice plate. It is found that the flow with constant velocity had an apposite influence slightly the dispersion at very short wavelengths and the ice profile was no longer aligned with the source velocity but rotated through an angle. Bhattacharjee and Sahoo [8] analyzed the interaction between current and flexural gravity waves generated due to a floating elastic plate under the assumptions of linearized theory and studied the effect of current on the wavelength, phase velocity, and group velocity of the flexural gravity waves in detail. Bhattacharjee and Sahoo [9] extended their study [8] to the generation of flexural gravity waves resulting from initial disturbances at a point and derived asymptotic depressions for the transient flexural gravity for large distances and times by the application of the method of the stationary phase. Mohanty et al. [10] studied a combined effect of the uniform current and compressive force on time-dependent flexural gravity wave 
motion in both the cases of single and two-layer fluids and derived the integral forms of Greens function and the velocity potential by using the method of stationary phase. Lu and Yeung [11] studied unsteady hydroelastic waves generated by point loads in a uniform current and found the flexural gravity wave motion depended on the ratio of current speed to phase or group speeds. Wang et al. [12] considered nonlinear hydroelastic waves generated due to a floating elastic plate in an underlying uniform current and studied analytically the effects of the uniform current on the nonlinear hydroelastic waves.

All the aforementioned literatures were based on the assumption that the underlying current is uniform in the fluid and hence ignore the effects of the vorticity distribution. However, in many situations, the current velocity in the vertical direction is mostly nonuniform and the vorticity appears (e.g., wind-driven current and tidal current). Hydroelastic waves with a linear shear or constant-vorticity current were investigated by many authors. Bhattacharjee and Sahoo [13] analyzed the effect of a linear shear current on the propagation of flexural gravity waves is analyzed in the frame of linearized shallow-water theory and derived the reflection and transmission coefficients based on the conservation of energy flux and the continuity of the vertical deformation of the ice sheet. Gao et al. [14] studied hydroelastic solitary waves in the presence of a linear shear current in the limit of deep water, and traveling solitary waves on water of infinite depth were computed for different values of vorticity and new generalised solitary waves were discovered. Recently, Gao et al. [15] investigated hydroelastic waves on water of finite depth interacting with a linear shear current in inviscid flows and derive a nonlinear Schrodinger equation for quasimonochromatic wavetrains and discuss the various behaviors of the coefficient of the nonlinear term from the NLS at different parameter values by employing a conformal mapping technique.

It is noted that the homotopy analysis method (HAM) $[16,17]$, which does not rely on any small physical parameter, has been applied to solve analytically the problem of the nonlinear wave-current interaction. Cheng et al. [18] investigated a train of periodic deepwater waves propagating on a steady shear current with a vertical distribution of vorticity by using the HAM and analyzed the influences of an exponential shear current on a train of waves in detail. Cang et al. [19] extend Cheng et al.'s study [18] on the effect of a background shear flow on periodic water waves by introducing a new parameter which measures the depth of the background shear current, and the impact of the depth parameter on the wave phase speed, the velocity profiles, and the maximum wave height were given. These works encouraged us to apply the HAM to the complex nonlinear problem of hydroelastic interaction among a floating elastic plate, water waves, and exponential shear currents.

In this work, our aim is to obtain accurate analytic approximations of nonlinear hydroelastic waves generated due to a floating elastic plate in a current which decays exponentially with depth. The influences of the exponential shear current on the hydroelastic wave profile, the wave phase speed, and the horizontal velocity profile are investigated and discussed in detail with the aid of the HAM. The remainder of the paper is organized as follows: In Section 2 , the mathematical model of nonlinear hydroelastic interaction among a floating elastic plate, water waves, and exponential shear currents is formulated, and the DubreilJacotin transformation is introduced to reformulate the original moving boundary problem into a fixed one. In Section 3, we present the solution procedure and the approximation and iteration of solutions in the frame of the HAM. In Section 4 , the results of numerical calculations and the influence of the shear current are shown. Finally, concluding remarks are given in Section 5.

\section{Mathematical Description}

Consider an incompressible flow of an inviscid but rotational fluid with a two-dimensioned case, we choose Cartesian coordinates $o x z$ in which the $x$-axis coincides with the undisturbed fluid-plate interface, while the $z$-axis points vertically upward. The floating elastic plate extends to the infinity along the $x$-axis. When the traveling waves exist, we use the moving coordinates $(x-c t, z) \longrightarrow(x, z)$ to eliminate time from the fluid-plate region, where $c$ is the wave speed. The conservation of mass for a twodimensional incompressible fluid is

$$
\frac{\partial(u+U-c)}{\partial x}+\frac{\partial v}{\partial y}=0
$$

where $(u, v)$ are the motions related to wave-current interaction in the $(x, y)$ directions, $U$ is the mean $x$-directed current, and the wave speed $c$ appears as negative due to the translation of the coordinate axis. We introduced a stream function $\psi(x, z)$, which satisfies exactly

$$
\begin{aligned}
u+U-c & =-\frac{\partial \psi(x, z)}{\partial y}, \\
v & =\frac{\partial \psi(x, z)}{\partial x} .
\end{aligned}
$$

Substituting the stream function into the governing equation derived by Lamb [20], we denote the vorticity distribution $\Omega$ as

$$
\frac{\partial v}{\partial x}-\frac{\partial(u+U-c)}{\partial y}=\nabla^{2} \psi=-\Omega(\psi), \quad(z \leq \zeta(x)) .
$$

Here, we study the shear current decay exponentially with depth, and let $\Omega(\psi)=\mu \exp (-\psi)$, where $\mu$ is a physical parameter determining the strength of the fluid vorticity. When $\mu<0$, the current moves in the same direction of wave propagation and then is called the aiding current. When $\mu>0$, the current is called the opposing current which moves in the opposing direction of wave propagation [21]. 


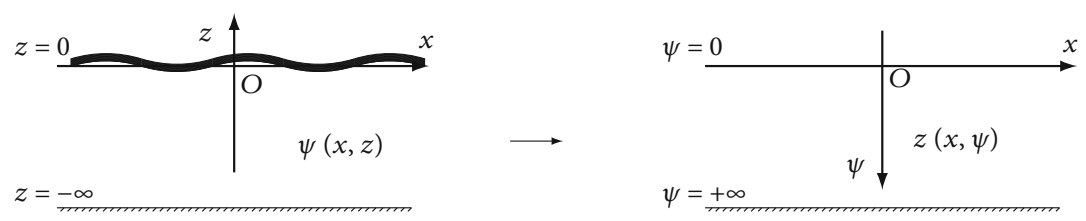

FIGURE 1: The coordinate transformation.

The impermeable bottom condition at deep water can be written as

$$
\frac{\partial \psi}{\partial x}=0, \quad(z=-\infty)
$$

With the assumption that there is no cavitation between the fluid and the plate with on draft, the unknown fluidplate interface $z=\zeta(x)$ is a streamline. Without loss of generality, the kinematic boundary conditions on $z=\zeta(x)$ can be written as

$$
\psi=0 \text {. }
$$

The nonlinear dynamic boundary conditions on the unknown fluid-plate interface streamline is described as

$$
\frac{1}{2}|\nabla \psi|^{2}+\frac{p_{e}}{\rho}+g \zeta=Q
$$

where $p_{e}$ is the pressure on the plate-water interface, $Q$ an unknown Bernoulli's constant on the fluid-plate interface streamline, $\rho$ is the uniform densities of the fluid, and $g$ is the gravitational acceleration.

As the surface pressure is equal to the pressure of the plate, we model the floating elastic plate as a linear Kirchhoff-Love plate [5].

$$
p_{e}=D \frac{\partial^{4} \zeta}{\partial x^{4}}+m_{e} g
$$

where the flexural rigidity of the plate is expressed by $D=E d^{3} /\left[12\left(1-v^{2}\right)\right]$ with Young's modulus $E$, the constant thickness $d$, and Poisson's ratio $v$ of the plate, respectively. $m_{e}\left(=\rho_{e} d\right)$ is the mass of the plate in a unit length with the uniform densities $\rho_{\mathrm{e}}$ of an elastic plate. Substituting Equation (7) into Equation (6) yields the full form of the dynamic boundary condition as

$$
\frac{1}{2}|\nabla \psi|^{2}+g \zeta+\frac{1}{\rho}\left(D \frac{\partial^{4} \zeta}{\partial x^{4}}+m_{e} g\right)=Q .
$$

It is difficult to directly solve the above Equations (3), (4), (5), and (8) in which the boundary conditions (5) and (8) satisfy the unknown fluid-plate interface $z=\zeta(x)$. So we use the Dubreil-Jacotion transformation $[18,19,21]$ to convert Cartesian coordinates oxz into Cartesian coordinates $o x \psi$ in which the $z$-axis points vertically downward, and then, the unknown interface $z=\zeta(x)$ is reformulated as the fixed location $\psi=0$, as shown in Figure 1 . Here, we consider that $z(x, \psi)$ is a periodic function in the $x$ direction with the period $2 \pi$.

For the sake of clarity, we introduce the following dimensionless quantities

$$
\begin{aligned}
& x^{*}=k x, z^{*}=k z, \psi^{*}=\frac{k}{c} \psi, \Omega^{*}(\psi)=\frac{\Omega(\psi)}{(k c)}, m_{e}^{*}=\frac{k m_{e}}{\rho}, \\
& \rho_{e}^{*}=\frac{\rho_{e}}{\rho}, D^{*}=k^{4} \frac{D}{(\rho g)}, E^{*}=\frac{k E}{(\rho g)}, H^{*}=k H,
\end{aligned}
$$

where variables with $*$ are dimensionless. By the DubreilJacotion transformation and the nondimensionalization (9), Equations (3), (8), and (4) are reformulated as (after omitting the $*$ )

$$
\begin{gathered}
\frac{\partial^{2} z}{\partial x^{2}}\left(\frac{\partial z}{\partial \psi}\right)^{2}-2 \frac{\partial z}{\partial x} \frac{\partial z}{\partial \psi} \frac{\partial^{2} z}{\partial x \partial \psi}+\left[1+\left(\frac{\partial z}{\partial x}\right)^{2}\right] \frac{\partial^{2} z}{\partial \psi^{2}} \\
=\left(\frac{\partial z}{\partial \psi}\right)^{3} \Omega(\psi), \quad(\psi>0), \\
\frac{1}{2} \delta\left[1+\left(\frac{\partial z}{\partial x}\right)^{2}\right]+\left(z+D \frac{\partial^{4} z}{\partial x^{4}}+m_{e}-\kappa\right)\left(\frac{\partial z}{\partial \psi}\right)^{2}=0, \quad(\psi=0),
\end{gathered}
$$

$$
\frac{\partial z}{\partial x}=0, \quad(\psi=+\infty)
$$

respectively, where both $\delta=c^{2} / c_{0}^{2}$ and $\kappa=k Q / g$ are unknown constants, and $c_{0}^{2}=g / k$ is known linear phase speed without any background current.

\section{Analytical Approach Based on the HAM}

3.1. Solution Expressions. From physical points of view, our hydroelastic problem is made of a train of deepwater hydroelastic waves, a uniform current due to the moving coordinates, and shear currents with exponential decay on depth. In case of the pure deepwater hydroelastic waves, the periodic wave deflection can be expressed by

$$
z=\sum_{m=0}^{+\infty} \alpha_{m} \cos (m x)
$$


where $\alpha_{m}$ is an unknown coefficient to be derived [5]. Considering the shear currents with vorticity distribution $\Omega(\psi)=\mu \exp (-\psi)$, Equation (10) contains the term exp $(-\psi)$, so it is suitable that $z(x, \psi)$ should contain the term $\exp (-n \psi)$, where $n$ is an integer. As hydroelastic wave deflections with shear currents are still periodic in the $x$ direction, then $z(x, \psi)$ should also contain the term $\cos (m x)$. Besides, the uniform currents caused by the coming coordinates do not give rise to the interaction between the hydroelastic waves and the currents. So we consider the solution expression of the hydroelastic wave deflection as

$$
z(x, \psi)=-\psi+\sum_{n=0}^{+\infty} \sum_{m=0}^{+\infty} \alpha_{m, n} \exp (-n \psi) \cos (m x)
$$

where $\alpha_{m, n}$ is an unknown coefficient to be derived.

According to the solution expressions (14), we may construct the initial estimation of the hydroelastic wave deflection as

$$
z_{0}(x, \psi)=-\psi+\frac{H}{2} \exp (-\psi) \cos (x)
$$

where $H$ is an unknown dimensionless wave height to be derived [18].

3.2. Deformation Equations. We construct three homotopies $Z(x, \psi ; q), \Delta(q)$, and $\Gamma(q)$. These homotopies are governed by the following zeroth-order deformation equations for the governing equation (10) and two boundary conditions (11) and (12) as

$$
(1-q) \mathscr{L}_{1}\left[Z(x, \psi ; q)-z_{0}(x, \psi)\right]=q c_{0} N_{1}[Z(x, \psi ; q)], \quad(\psi>0),
$$

$(1-q) \mathscr{L}_{2}\left[Z(x, \psi ; q)-z_{0}(x, \psi)\right]=q c_{0} N_{2}[Z(x, \psi ; q), \Delta(q), \Gamma(q)]$, $(\psi=0)$,

$$
\frac{\partial Z(x, \psi ; q)}{\partial x}=0, \quad(\psi=+\infty)
$$

respectively, with the wave height

$$
Z(0,0 ; q)-Z(\pi, 0 ; q)=H
$$

where $q \in[0,1]$ is the embedding parameter. When $q$ increases from 0 to $1, Z(x, \psi ; q)$ varies continuously from its initial estimation $z_{0}(x, \psi)$ to the exact solution $z(x, \psi), \Delta$ (q) deforms continuously from its initial estimation $\delta_{0}$ to the exact solution $\delta$, and $\Gamma(q)$ is from $\kappa_{0}$ to $\kappa . c_{0}$ is a nonzero convergence-control parameter. Based on the governing

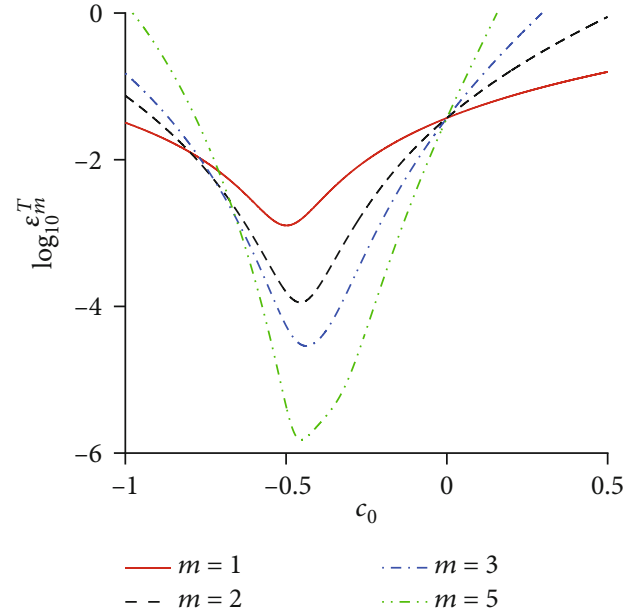

FIgURE 2: Residual squares of $\log _{10} \varepsilon_{m}^{T}$ of the $m$ th order homotopy approximation $c_{0}$.

equation (10) and the boundary condition (11), $\mathcal{N}_{1}[\cdot]$ and $\mathcal{N}_{2}[\cdot]$ are the nonlinear operators defined by

$$
\begin{gathered}
\mathcal{N}_{1}[Z(x, \psi ; q)]=\frac{\partial^{2} Z}{\partial x^{2}}\left(\frac{\partial Z}{\partial \psi}\right)^{2}-\frac{\partial Z}{\partial x} \frac{\partial Z}{\partial \psi} \frac{\partial^{2} Z}{\partial x \partial \psi} \\
+\left[1+\left(\frac{\partial Z}{\partial x}\right)^{2}\right] \frac{\partial^{2} Z}{\partial \psi^{2}}-\left(\frac{\partial Z}{\partial \psi}\right)^{3} \Omega(\psi), \\
\mathcal{N}_{2}[Z(x, \psi ; q), \Delta(q), \Gamma(q)]=\frac{1}{2} \Delta\left[1+\left(\frac{\partial Z}{\partial x}\right)^{2}\right] \\
+\left(Z+D \frac{\partial^{4} Z}{\partial x^{4}}+m_{e}-\Gamma\right)\left(\frac{\partial Z}{\partial \psi}\right)^{2},
\end{gathered}
$$

respectively.

If we only choose the unique linear term $\partial^{2} / \partial \psi^{2}$ in the equation (10) as the auxiliary linear operators $\mathscr{L}_{1}$, we would get a solution $z(x, \psi)$ in the power series of $\psi$ which cannot satisfy the impermeable bottom condition (12). We can obey the solution expression (14) under the physical considerations to choose the following auxiliary linear operator

$$
\mathscr{L}_{1}[u]=\frac{\partial^{2} u}{\partial \psi^{2}}+\frac{\partial^{2} u}{\partial x^{2}}
$$

where $\mathscr{L}_{1}[0]=0$.

The nonlinear boundary condition (11) does not contain any linear term. Here, we still follow the solution expression (14) to choose another auxiliary linear operator

$$
\mathscr{L}_{2}[u]=u+\frac{\partial u}{\partial \psi}
$$

where $\mathscr{L}_{2}[0]=0$. 


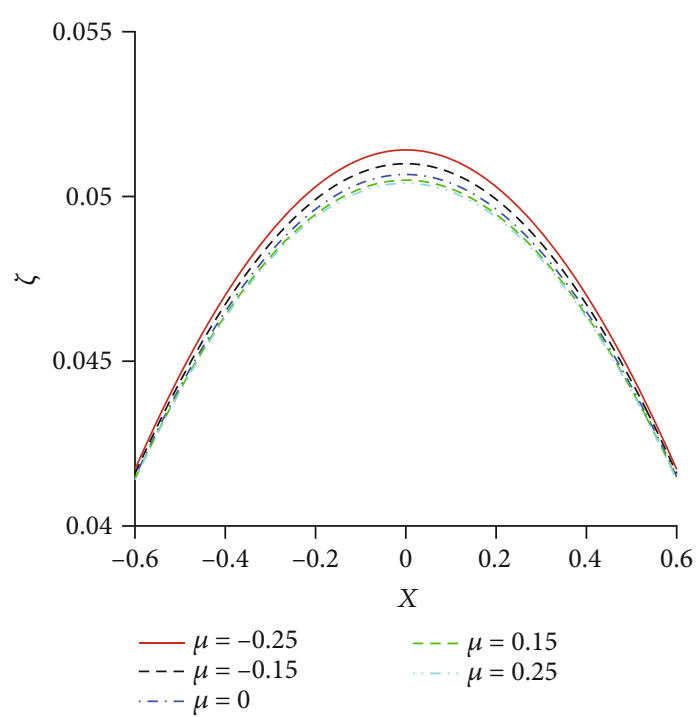

(a) Wave elevations near the crest

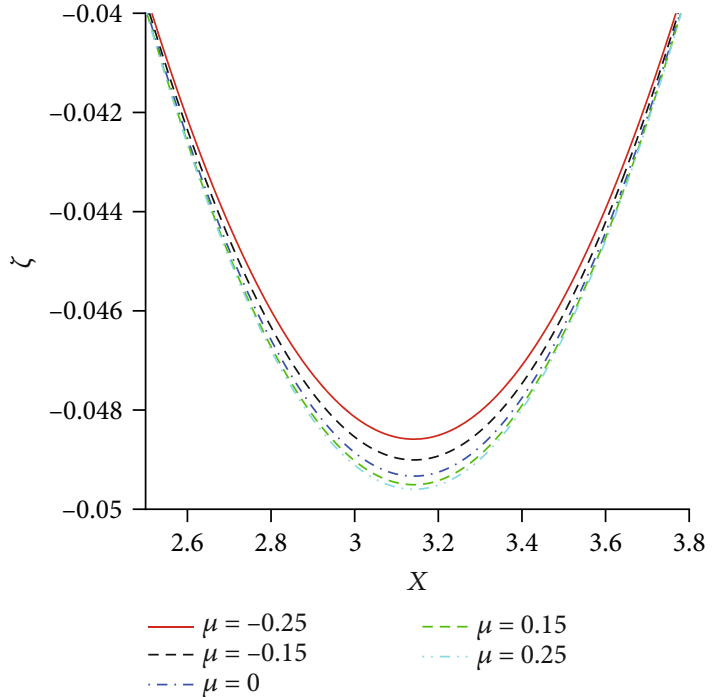

(b) Wave elevations near the trough

FIgURE 3: Variation of the hydroelastic wave profiles versus $x$ for different vorticity parameter $\mu$.

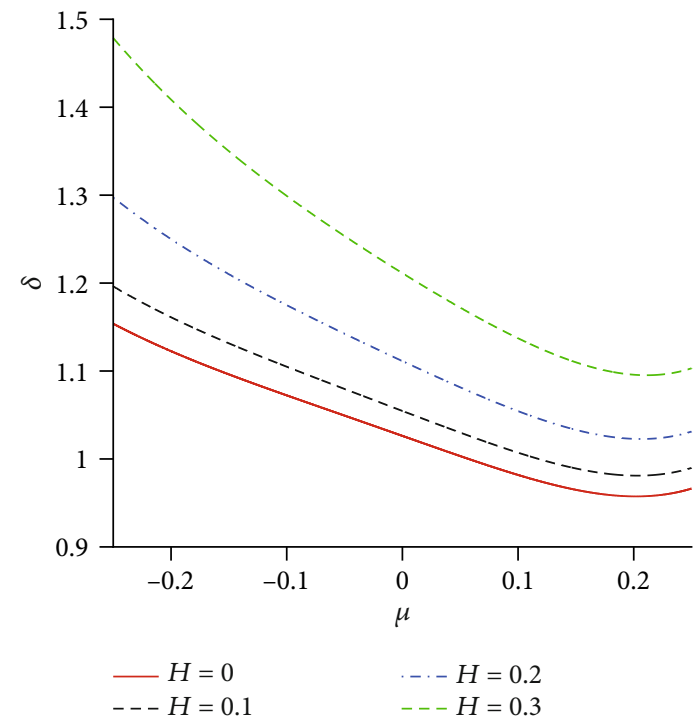

FIGURE 4: The influence of the vorticity parameter $\mu$ on the phase velocity $\delta\left(=c^{2} / c_{0}^{2}\right)$ for different wave amplitude $H$.

Expanding the unknown function $Z(x, \psi ; q)$ and the two unknown constants $\Delta(q)$ and $\Gamma(q)$ into the Maclaurin series about $q$ at $q=0$,

$$
\begin{aligned}
Z(x, \psi ; q) & =\sum_{m=0}^{+\infty} z_{m}(x, \psi) q^{m}, \\
\Delta(q) & =\sum_{m=0}^{+\infty} \delta_{m} q^{m}, \\
\Gamma(q) & =\sum_{m=0}^{+\infty} \kappa_{m} q^{m},
\end{aligned}
$$

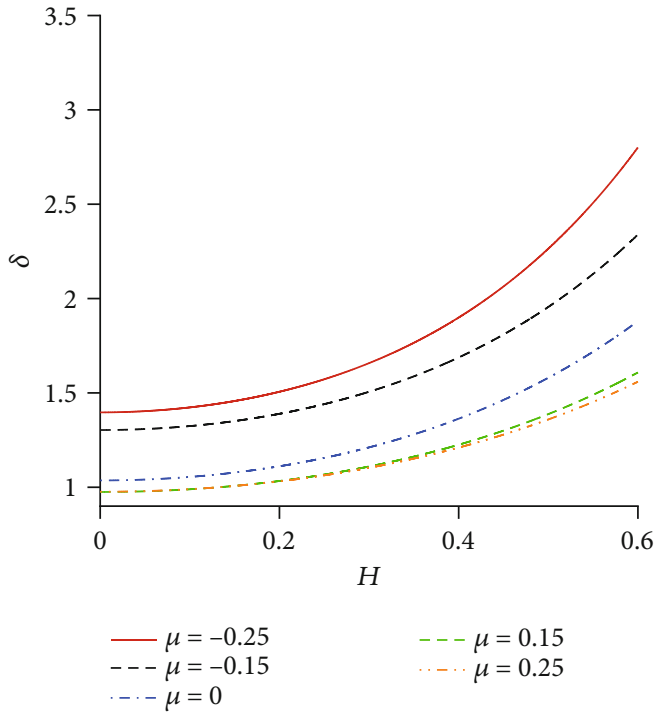

Figure 5: The influence of the wave amplitude $H$ on the phase velocity $\delta\left(=c^{2} / c_{0}^{2}\right)$ for different vorticity parameter $\mu$.

where

$$
\left\{z_{m}(x, \psi), \delta_{m}, \kappa_{m}\right\}=\left.\frac{1}{m !} \frac{\partial^{m}}{\partial q^{m}}\{Z(x, \psi ; q), \Delta(q), \Gamma(q)\}\right|_{q=0} .
$$

We substitute these series (23), (24), and (25) into the zeroth-order deformation equations (16) and (17) and differentiate the zero-order deformation equations $m$ times about $q$, then divide them by $m$ !. Setting $q=0$, we can obtain the linear PDEs (i.e., high-order deformation equations in the 


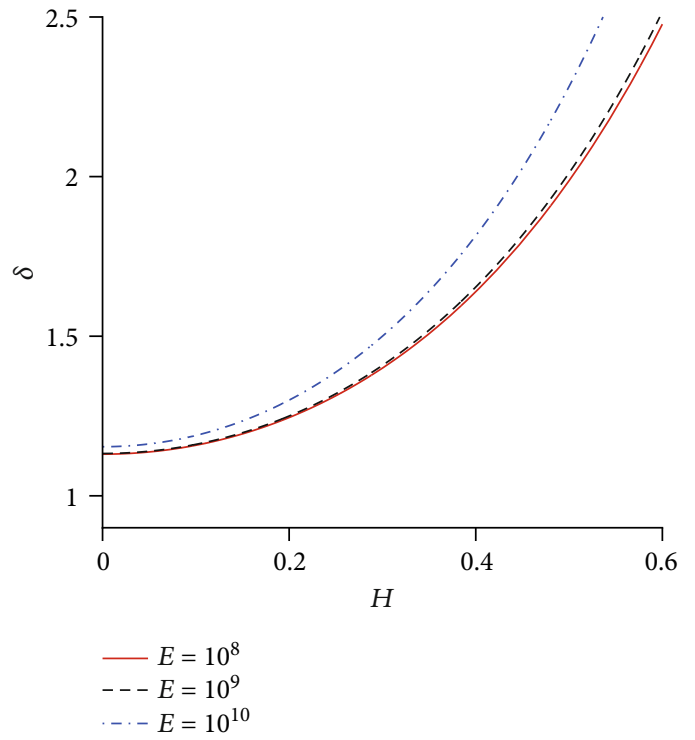

(a) $\mu=-0.2$

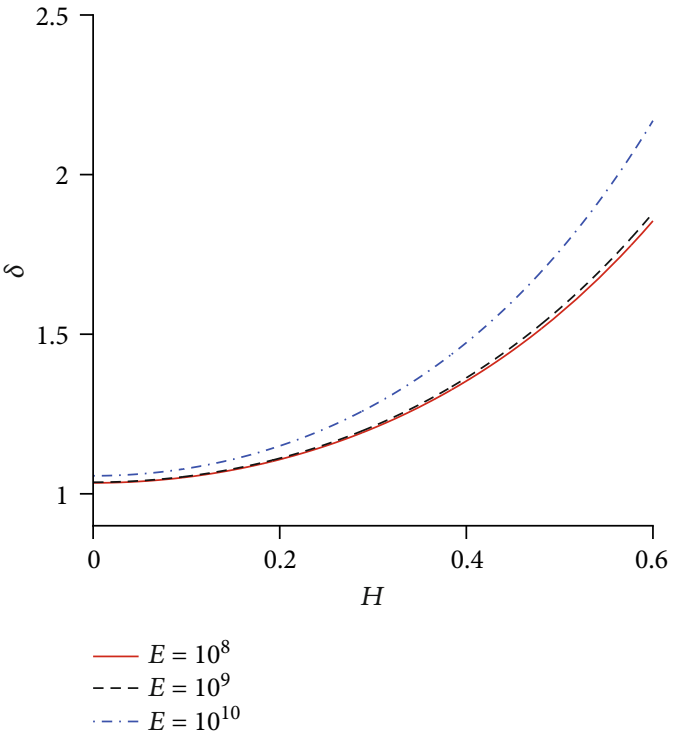

(b) $\mu=0$

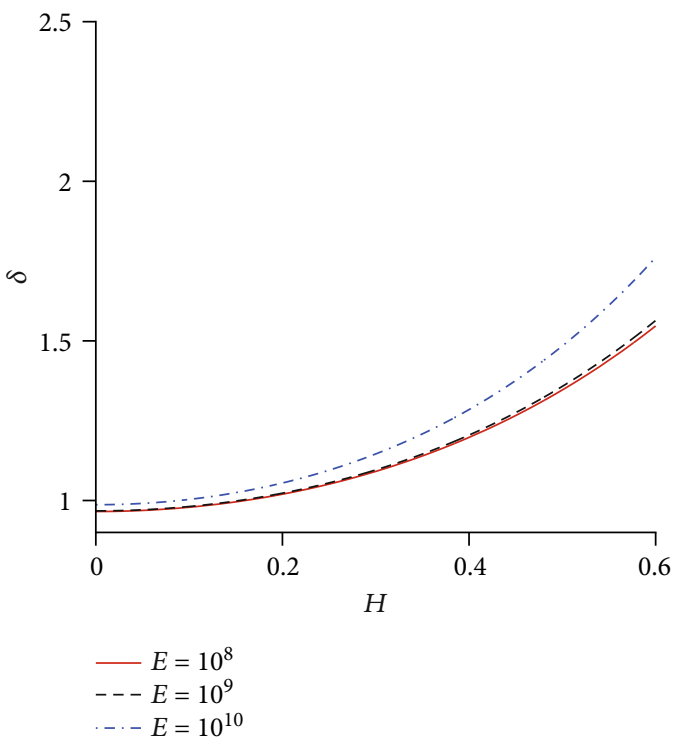

(c) $\mu=0.2$

Figure 6: The influence of Young's modulus $E$ on the phase velocity $\delta\left(=c^{2} / c_{0}^{2}\right)$ for different wave amplitude $\mu$.

HAM) for the unknown function $z_{m}(x, \psi)$ and the unknown constants $\delta_{m}$ and $\kappa_{m}$. In order to make our equations closed, we consider

$$
z_{n}(0,0)-z_{n}(\pi, 0)=H, \quad(n \geq 0)
$$

to relate the solutions and the wave height $H$.

3.3. Optimal Convergence-Control Parameters. To ensure the accuracy of our HAM-based series solutions, we define the total squared error $\varepsilon^{T}$ as follows:

$$
\varepsilon_{m}^{T}=\varepsilon_{m, 1}+\varepsilon_{m, 2},
$$

where

$$
\begin{aligned}
\varepsilon_{m, 1} & =\frac{1}{(1+M)^{2}} \sum_{i=0}^{M} \sum_{j=0}^{M}\left(\left.\mathcal{N}_{1}[Z(x, \psi ; q)]\right|_{x=i \Delta x, \psi=j \Delta \psi}\right)^{2}, \\
\varepsilon_{m, 2} & =\frac{1}{1+M} \sum_{i=0}^{M}\left(\left.\mathcal{N}_{2}[Z(x, \psi ; q), \Delta(q), \Gamma(q)]\right|_{x=i \Delta x, \psi=0}\right)^{2},
\end{aligned}
$$

where $\varepsilon_{m, 2}$ are the residual square errors of Equations (10) and (11), respectively. $\Delta x=\Delta \psi=\pi / M$. In this paper, we choose $M=10$. The optimal convergence-control parameter $c_{0}$ can be acquired by the minimum value of $\varepsilon_{m}^{T}$ in the residual plot as shown in Figure 2 


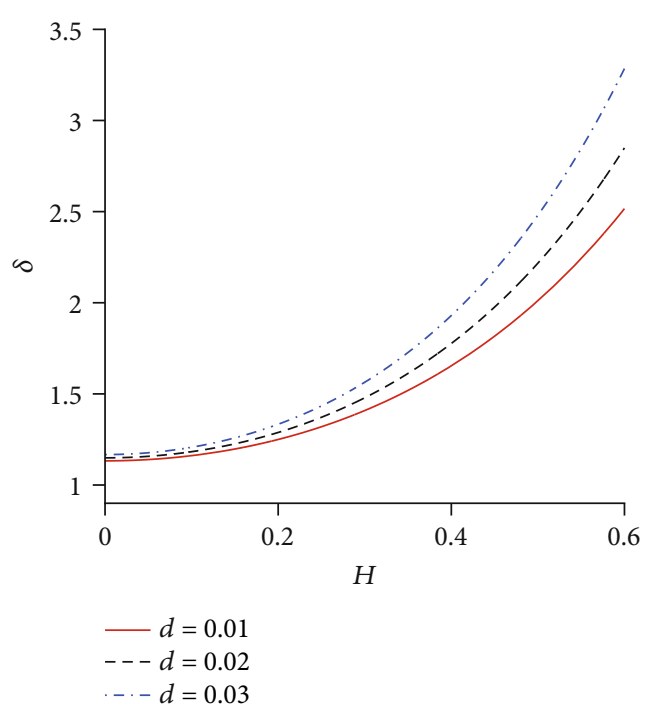

(a) $\mu=-0.2$

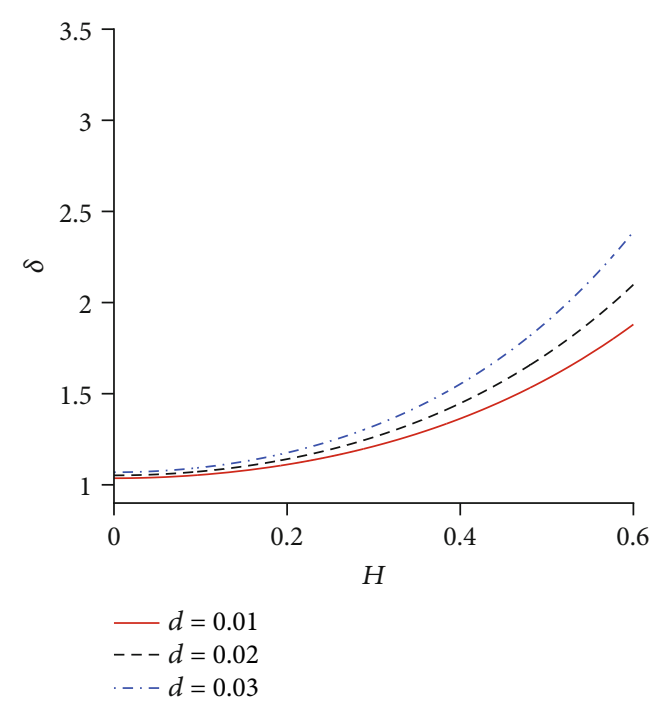

(b) $\mu=0$

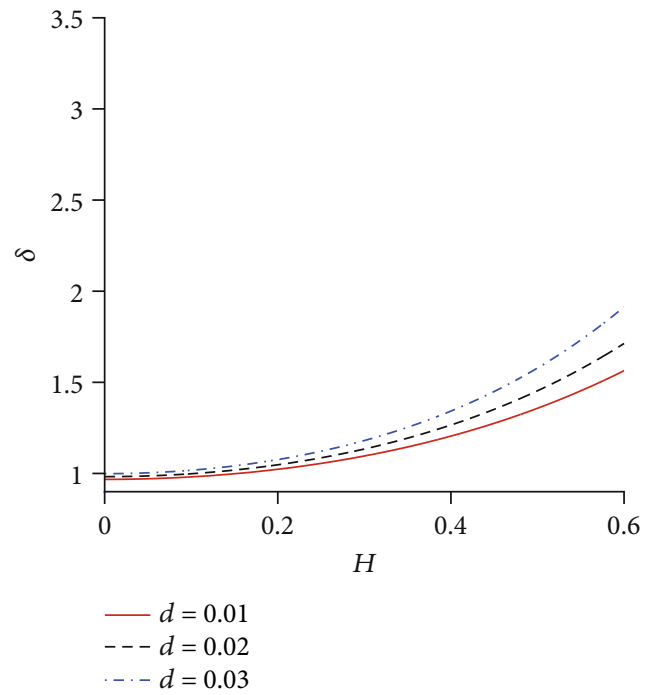

(c) $\mu=0.2$

FIgURE 7: The influence of the plate's thickness $d$ on the phase velocity $\delta\left(=c^{2} / c_{0}^{2}\right)$ for different wave amplitude $\mu$.

3.4. Iteration of Solutions. Substituting the initial estimation of hydroelastic wave deflection (15) into the high-order deformation equations, we can obtain every order analytic solution from these deformation equations by performing symbolic computation using Mathematica 8.0. First, oneorder solution for the unknown function $z(x, \psi)$ is acquired from the one-order deformation equations as follows:

$$
\begin{aligned}
& z_{1}(x, \psi)=\left[\mu \exp (-\psi)-\frac{1}{4} H^{2} \exp (-2 \psi)+\frac{3}{8} H^{2} \mu \exp (-3 \psi)\right] c_{0} \\
& +\left[\left(-1-\frac{1}{2} D-\frac{1}{2} \delta_{0}+\kappa_{0}-\frac{3}{32} H^{2}-\frac{3}{32} D H^{2}\right) \exp (-\psi)\right. \\
& -\frac{3}{2} \mu \exp (-2 \psi)+\frac{1}{16} H^{2} \exp (-3 \psi) \\
& \left.-\frac{3}{32} H^{2} \mu \exp (-4 \psi)\right] c_{0} H \cos t(x)
\end{aligned}
$$

$$
\begin{aligned}
& +\left[\left(-\frac{5}{24}-\frac{1}{8} D-\frac{1}{16} m_{e}-\frac{1}{32} \delta_{0}+\frac{1}{16} \kappa_{0}\right)\right. \\
& \left.+\left(\frac{1}{24}-\frac{1}{8} \mu\right) \exp (-\psi)+\frac{1}{16} \exp (-2 \psi)\right] c_{0} H^{2} \\
& \times \exp (-2 \psi) \cos (2 x)+\left[-\left(\frac{7}{384}+\frac{1}{96} D\right)-\frac{1}{256} \mu \exp (-\psi)\right. \\
& \left.+\frac{1}{384} \exp (-2 \psi)+\frac{1}{768} \mu \times \exp (-3 \psi)\right] c_{0} H^{3} \exp (-3 \psi) \cos (3 x),
\end{aligned}
$$

where the initial solutions $\delta_{0}$ of $\delta$ and $\kappa_{0}$ of $\kappa$ are still unknown. We use the relation (27) for the wave amplitude and the vertical displacement to determine $\delta_{0}$ and $\kappa_{0}$ as follows: 


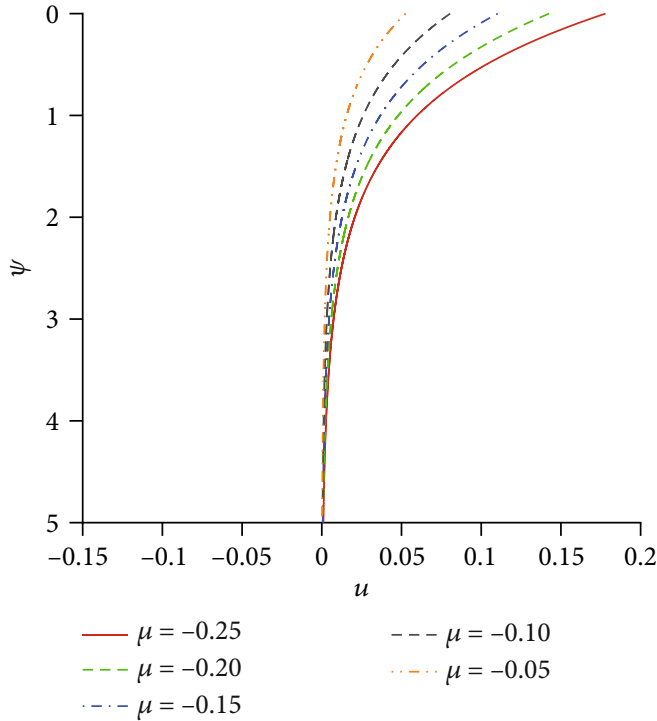

(a) Horizontal velocity $u$ near the crest

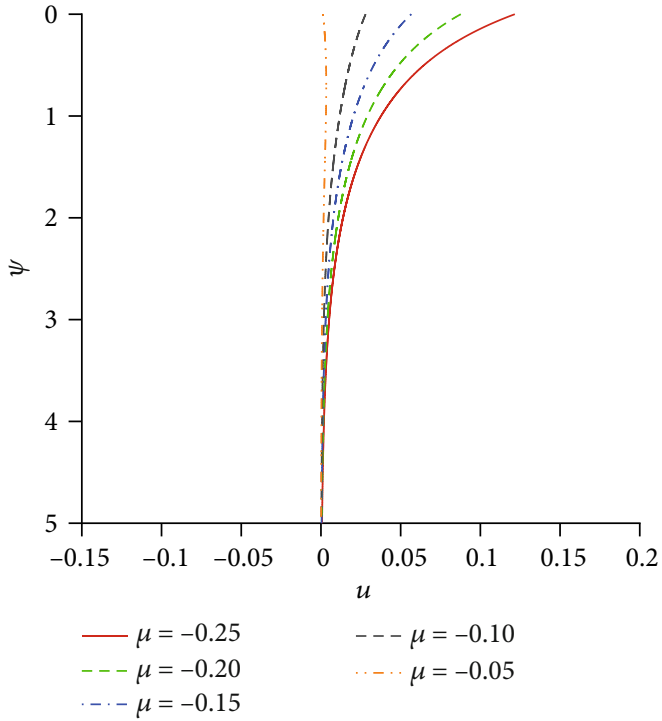

(b) Horizontal velocity $u$ near the trough

Figure 8: The influence of the aiding shear currents on horizontal velocity profile $u$ of propagating periodic waves for different vorticity parameter $\mu$ by means of $c_{0}=-0.4$. Solid line: $\mu=-0.25$; dashed line: $\mu=-0.20$; dash dot line: $\mu=-0.15$; long dash line: $\mu=-0.10$; dash dot dot: $\mu=-0.05$.

$$
\begin{aligned}
\delta_{0}= & 2+\frac{1}{c_{0}}+D\left(1-\frac{H^{2}}{6}+\frac{5 H^{4}}{192}\right)+\mu\left(1-\frac{35 H^{2}}{192}+\frac{37 H^{4}}{1536}\right) \\
& +\frac{3 c_{0}+32}{256 c_{0}} H^{4}+\frac{11}{32} H^{2}, \\
\kappa_{0}= & 2+\frac{1}{c_{0}}+D\left(1-\frac{H^{2}}{48}+\frac{5 H^{4}}{384}\right)+\mu\left(2+\frac{H^{2}}{192}+\frac{37 H^{4}}{3072}\right) \\
& +m_{e} \frac{7 c_{0}+2}{32 c_{0}} H^{2}+\frac{3}{512} H^{4},
\end{aligned}
$$

respectively. Once the convergence control parameter $c_{0}$ and important physical parameters $H, D, \mu$, and $m_{\mathrm{e}}$ are given, we can get the corresponding solutions for our hydroelastic problem. If we continue with the one-order approximations by utilizing the high-order deformation equations, the higher-order approximations can be acquired iteratively.

\section{Result Analysis}

First, we illustrate the total squared residual error $\varepsilon_{m}^{T}$ of our solutions at several different orders versus $c_{0}$ with the case of $\mu=0.1, H=0.1, d=0.01, \rho_{\mathrm{e}}=0.9, E=12822.8$ (i.e., the dimensional $E=109 \mathrm{~Pa}$ ), and $\nu=0.33$ and take these data hereinafter for computation unless otherwise stated. As shown in Figure 2, we find that $\varepsilon$ in Equation (28) decreases firstly and then increases in the interval $[-1.0,0]$. And as the order $m$ increases, $\varepsilon$ decreases gradually about -0.4 . Then, the optimal value of $c_{0}$ can be chosen as -0.4 . This illustrates that our HAM-based series solutions are accurate and convergent for the nonlinear hydroelastic interaction.

The plate deflections at the crest and the trough in the case of $H=0.1$ and $-0.25 \leq \mu \leq 0.25$ are shown in Figures 3(a) and 3(b), respectively. It is found that, for a given wave height $H$, the aiding exponential shear current $(\mu<0)$ tends to sharpen the crest and smoothen the trough, while the opposing shear current $(\mu>0)$ has the opposite effect. And the effect of the shear current on plate deformation is more obvious at the trough than at the crest. This might explain why the aiding exponential shear current tends to shorten the maximum wave height while the opposing one tends to enlarge it.

In Figure 4, we show a fourth-order dispersion relation for the phase speed $\delta\left(=c^{2} / c_{0}^{2}\right)$ as a function of the vorticity parameter $\mu$ with several given wave heights $H$. And Figure 5 shows the phase speed $\delta\left(=c^{2} / c_{0}^{2}\right)$ as a function of the wave heights $H$ with several given vorticity parameter $\mu$. It is found that, for both an opposing current and an aiding current, the larger value of the wave height increases the wave phase speed, while the phase speed decreases with the increase of the vorticity parameter in the opposing current, but the phase speed increases with the increase of the vorticity parameter in the aiding current. As shown in Figures 4 and 5 , the phase speed $\delta$ is close to 1 when $H$ is very small (linear waves) and $\mu$ (no current). It is demonstrated that our result is compatible with the dispersion relation in deep water $\delta=(1+D) /(1+m)$ [22] from the linearized theory of hydroelastic waves with no current.

The effects of Young's modulus of the plate are shown in Figure 6, from which we can see that for both an opposing current and an aiding current, the phase speed $\delta$ increases with increasing Young's modulus. The effects of the plate 


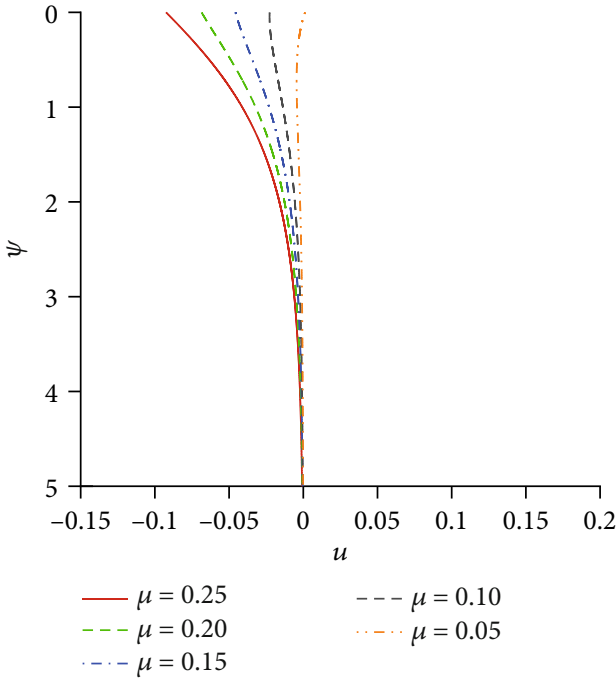

(a) Horizontal velocity $u$ near the crest

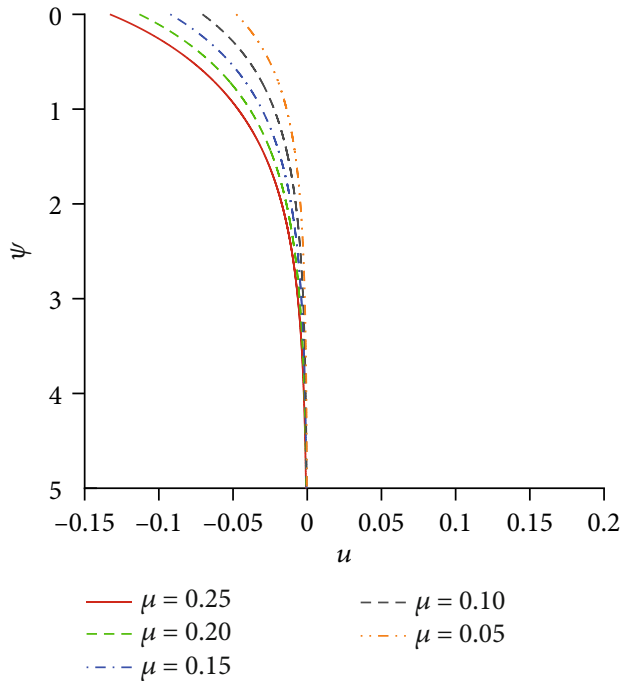

(b) Horizontal velocity $u$ near the trough

FIGURE 9: The influence of the opposing shear currents on the horizontal velocity profile $u$ of propagating periodic waves for different vorticity parameter $\mu$ by means of $c_{0}=-0.4$. Solid line: $\mu=0.25$; dashed line: $\mu=0.20$; dash dot line: $\mu=0.15$; long dash line: $\mu=0.10$; dash dot dot: $\mu=0.05$.

thickness $d$ on the phase speed $\delta$ are studied. Figure 7 shows that a larger $d$ increases the phase speed, which is similar to effects for Young's modulus of the plate in Figure 6.

Figure 8 shows the horizontal velocity profiles $u(=1+$ $\left.z_{\psi}(x, \psi)^{-1}\right)$ in the aiding shear currents for different vorticity parameters $\mu$. We find that the horizontal velocity under the wave crest is in the same direction, while the horizontal velocity under the wave trough changes from the same direction into the opposing direction when the absolute value of $\mu$ decreases to about 0.05 . Figure 9 shows the horizontal velocity profiles in the opposing shear currents for different vorticity parameters $\mu$. We observe that the horizontal velocity under the wave trough is in the opposing direction, while the horizontal velocity under the wave crest changes from the opposing direction into the same direction when the value of $\mu$ decreases to about 0.05 . As shown in Figures 8 and 9, we observe that for both an opposing current and an aiding current, a larger $\mu$ tends to increase the horizontal velocity $u$. Besides, in the opposing current, the horizontal velocity $u$ under the wave crest delays more quickly as $\psi$ increases than that of waves under the wave trough, while in the aiding current case, we observe the opposite effect.

\section{Conclusions}

In this work, we are concerned with nonlinear hydroelastic waves generated due to a floating elastic plate interacting with a shear current which decays exponentially with depth. We introduce a stream function to obtain the governing equation with the boundary conditions expressing a balance among the hydrodynamic, the shear currents, elastic, and inertial forces. In order to simplify the algorithm, we transfer the nonlinear boundary value problem from an unknown free surface into a known boundary by means of DubreilJacotin transformation. In the frame of the HAM, we consider the solution expression of the hydroelastic wave deflection as a series with a set of base functions $\exp (-n \psi)$ $\cos (m x)$ based on physical points of view. Numerical results demonstrate the validity and convergence of our HAM-based analytical solutions for the nonlinear hydroelastic interaction a wave-current coexisting fluid.

The effects of some important physical parameters on the plate deflections, the phase speed, and the horizontal velocity profiles are considered in detail. We find that a larger aiding shear current tends to sharpen the crest and smoothen the trough of the plate deflections, while the opposing shear current has the opposite effect, and both opposing and aiding current on plate deformation all have a more obvious effect on the trough than on the crest.

For both an opposing current and an aiding current, the larger wave height $H$ increases the wave phase speed $\delta$, while the aiding exponential shear currents tend to increase the wave phase velocity, but the opposing exponential shear currents tend to decrease it. It is noted that our result is compatible with the dispersion relation in deep water from the linearized theory of hydroelastic waves with no current.

The horizontal velocity under a shear current is in the same direction, while the velocity's direction changes as the value of vorticity parameters $\mu$ decreases. But in the case of an opposing current, the horizontal velocity under the wave crest delays more quickly as $\psi$ increases than that of waves under the wave trough, while in the aiding current case, there is an opposite effect. All of those results obtained here can help us further understand hydroelastic interaction between a floating elastic plate and wave current in the real ocean. 


\section{Data Availability}

All models generated or used during the study appear in the submitted article. All code generated or used during the study are available from the corresponding author by request (Ping Wang, Email: pingwang2003@126.com).

\section{Conflicts of Interest}

There is no any conflict of interests in our manuscript. The authors themselves program the symbolic computation software named Mathematica 8.0 independently to gain the approximate analytical solutions of the PDEs considered here.

\section{Acknowledgments}

This research was financially supported by the Natural Science Foundation of Shandong Province under Grant No. ZR2017MA014 and the National Natural Science Foundation of China under Grant No. 51674149. Qingdao Postdoctoral Applied Research Project No. 020022034 is also acknowledged.

\section{References}

[1] L. K. Forbes, "Surface waves of large amplitude beneath an elastic sheet. Part 1. Highorder series solution," Journal of Fluid Mechanics, vol. 169, no. 1, pp. 409-428, 1986.

[2] L. K. Forbes, "Surface waves of large amplitude beneath an elastic sheet. Part 2. Galerkin solution," Journal of Fluid Mechanics, vol. 188, pp. 491-508, 1988.

[3] J.-M. Vanden-Broeck and E. I. Părău, "Two-dimensional generalized solitary waves and periodic waves under an ice sheet," Philosophical Transactions of the Royal Society A: Mathematical, Physical and Engineering Sciences, vol. 369, no. 1947, pp. 2957-2972, 2011.

[4] P. A. Milewski, J. M. Vanden-Broeck, and Z. Wang, "Hydroelastic solitary waves in deep water," Journal of Fluid Mechanics, vol. 679, pp. 628-640, 2011.

[5] P. Wang and D. Q. Lu, "Analytic approximation to nonlinear hydroelastic waves traveling in a thin elastic plate floating on a fluid," Science China Physics, Mechanics and Astronomy, vol. 56, no. 11, pp. 2170-2177, 2013.

[6] P. Wang and D. Q. Lu, "Nonlinear hydroelastic waves traveling in a thin elastic plate floating on a two-layer fluid," Applied Mathematics and Computation, vol. 274, pp. 700-710, 2016.

[7] R. Schulkes, R. Hosking, and A. Sneyd, "Waves due to a steadily moving source on a floating ice plate. Part 2," Journal of Fluid Mechanics, vol. 180, no. 1, pp. 297-318, 1987.

[8] J. Bhattacharjee and T. Sahoo, "Interaction of current and flexural gravity waves," Ocean Engineering, vol. 34, no. 11-12, pp. 1505-1515, 2007.

[9] J. Bhattacharjee and T. Sahoo, "Flexural gravity wave generation by initial disturbances in the presence of current," Journal of Marine Science and Technology, vol. 13, no. 2, pp. 138-146, 2008.

[10] S. Mohanty, R. Mondal, and T. Sahoo, "Time dependent flexural gravity waves in the presence of current," Journal of Fluids and Structures, vol. 45, pp. 28-49, 2014.
[11] D. Q. Lu and R. W. Yeung, "Hydroelastic waves generated by point loads in a current," International Journal of Offshore and Polar Engineering, vol. 25, no. 1, pp. 8-12, 2015.

[12] P. Wang, Y. Wang, C. Su, and Y. Yang, "Nonlinear hydroelastic waves generated due to a floating elastic plate in a current," Advances in Mathematical Physics, vol. 2017, Article ID 2837603, 9 pages, 2017.

[13] J. Bhattacharjee and T. Sahoo, "Interaction of flexural gravity waves with shear current in shallow water," Ocean Engineering, vol. 36, no. 11, pp. 831-841, 2009.

[14] T. Gao, P. Milewski, and J.-M. vanden-Broeck, "Hydroelastic solitary waves with constant vorticity," Wave Motion, vol. 85, pp. 84-97, 2019.

[15] T. Gao, Z. Wang, and P. Milewski, "Nonlinear hydroelastic waves on a linear shear current at finite depth," Journal of Fluid Mechanics, vol. 876, pp. 55-86, 2019.

[16] S. J. Liao, Beyond Perturbation: Introduction to the Homotopy Analysis Method, CRC Press, Boca Raton, 2004.

[17] S. J. Liao, Homotopy Analysis Method in Nonlinear Differential Equations, Higher Education Press, Beijing, 2012.

[18] J. Cheng, J. Cang, and S. Liao, "On the interaction of deep water waves and exponential shear currents," Zeitschrift für angewandte Mathematik und Physik, vol. 60, no. 3, pp. 450478, 2009.

[19] J. Cang, J. Cheng, and R. Grimshaw, "A short comment on the effect of a shear layer on nonlinear water waves," Science China Physics, Mechanics \& Astronomy, vol. 54, no. 1, pp. 67-73, 2011.

[20] H. Lamb, Hydrodynamics, Dover Press, 6th edition, 1945.

[21] T. Wang and G. C. Li, "Effect of nonlinear wave-current interaction on flow fields and hydrodynamic forces," Science in China Series A: Mathematics, vol. 40, no. 6, pp. 622-632, 1997.

[22] F. Xu and D. Q. Lu, "An optimization of eigenfunction expansion method for the interaction of water waves with an elastic plate," Journal of Hydrodynamics, vol. 21, no. 4, pp. 526-530, 2009. 